\title{
On Modeling of Tall Linear Systems with Multirate Outputs
}

\author{
Mohsen Zamani, Student Member, IEEE, Brian D.O. Anderson, Life Fellow, IEEE, Elisabeth Felsenstein, and \\ Manfred Deistler, Fellow, IEEE.
}

\begin{abstract}
Motivated by problems of modeling high dimensional time series, this paper considers time-invariant, discretetime linear systems which have a larger number of outputs than inputs, with the inputs being independent stationary white noise sequences. Moreover, different outputs are measured at different rates (in economic modeling, it is common that some variables are measured monthly and others quarterly). In particular, the paper focuses on the case where the number of measurements is extremely large compared to the number of inputs. In the current paper, our ultimate goal is to identify the parameter matrices of such systems from outputs covariance data. To achieve this main goal and avoid excessively high dimensionality in the model, we use the notion of static factor, which roughly is a special subvector of the latent vector i.e. those parts of output vector remaining after removal of contaminating additive noise in the measurement. Since the model associated with the static factor is periodic in the output parameters, we use the well-known technique of blocking to obtain a blocked linear time-invariant system associated with this model. It is illustrated that this blocked system is generically zero-free. Then we use the spectral factorization technique to obtain the parameter matrices associated with the blocked system. These parameter matrices can be obtained by a finite number of rational calculations from the spectral matrix due to the generic zero-freeness of tall spectral matrices. Finally, we use the parameter matrices associated with the blocked system to obtain the parameter matrices associated with the static factor and ultimately those of the original underlying unblocked system.
\end{abstract}

\section{INTRODUCTION}

In different applications, one has to deal with dynamic systems with a larger number of measured outputs than inputs. For instance, in generalized dynamic factor models (GDFMs) [1] which arise in the field of econometric modeling, usually the number of collected data sequences (measured scalar output processes in the systems and control terminology) is much larger than the number of shocks (scalar white noise input sequences in the systems and control terminology $)^{1}$. It is also very common in econometric modeling to measure outputs at different rates. For instance, some measured outputs may be obtained monthly while some others may be collected quarterly or even annually.

Proofs in this paper are omitted; they are available from the first author upon request and will be given in a full length version of this paper.

M. Zamani and B. D. O. Anderson are with Research School of Engineering, Australian National University, Canberra, ACT 0200, Australia. B. D. O. Anderson is with National ICT Australia (NICTA). \{mohsen. zamani, brian. anderson\}@anu. edu. au.

E. Felsenstein and M. Deistler are with Department of mathematical methods in Economics, Technical university of Vienna, 8/119 Argentinierstrasse, A 1040 Vienna, Austria. \{elisabeth. felsenstein, deistler\}atuwien.ac.at

${ }^{1}$ In this paper we mostly use the systems and control jargon.
Even though the authors of this work have been interested in properties of these systems due to their application in GDFMs, the paper does not focus on the application problem, but rather on the underlying system theoretic issues involved with tall multirate systems.

In the context of GDFMs, latent variables i.e. those parts of output data remaining after removal of contaminating additive noise in the measurement, are modeled by linear time-invariant finite-dimensional systems with unobserved white noise input. In the single-rate setting i.e. when all latent variables are available at the same rate, it is shown that these systems are generically zero-free [2], [3] and [4]. This means that the latent variables can be modeled as a singular autoregressive process whose parameters can be straightforwardly identified using Yule-Walker equations from covariance data [5].

In contrast to the single-rate case, the multirate case i.e. when one or more latent variables are provided at different rates, is more complicated. In reference [6], the authors use the blocking or lifting technique to study the zeros of blocked linear time-invariant systems associated with the underlying unblocked multirate system. The authors in [6] specify a situation under which the blocked system is generically zero-free. Then along the same lines, the authors of [7] study a regular autoregressive model operating at the highest sampling frequency and use the modified extended YuleWalker equations to identify the model parameters from the population second moments which can be observed in principle ${ }^{2}$. Note that the technique used in [7] is inspired from the method of extended Yule-Walker equations initially introduced in [8]. It is worthwhile mentioning that the results of [7] are also applicable to a class of singular autoregressive models. In [9], the authors study identifiability for another class of singular autoregressive systems.

The approaches discussed in [7] and [8] are effective, apart from possibly one issue. When the size of the measured outputs vector becomes extremely large, direct use of these approaches may not be practical for identifying the underlying system parameters from the covariance matrix associated with the latent variables. In an autoregressive model, for example, the coefficient matrices will each contain $l^{2}$ real parameters, where $l$ is the output dimension. This paper deals with the problem by showing how to use the notion of static factor to handle problems involved with having large cross-sectional dimension in the measurements

\footnotetext{
${ }^{2}$ Second order moments which are observed 'in principle' are those obtainable from sample statistics when the number of samples goes to infinity.
} 
when identifying the underlying system parameters from the output covariance data. Even though the static factor has been used for identifying the parameter matrices of the original underlying system when all measured outputs are available at all times i.e. single-rate case [5], the study of the minimal static factor in the multirate case is not a trivial extension of available results in the single-rate case. In the multirate case the latent vector consists of two parts, viz. the fast part and the slow part, with one stacked over the other. The static factor can be easily obtained from the latent vector through some linear operations and almost always has a much smaller dimension compared to the original output vector. In particular, in this paper we separately define the static factor for the fast and the slow parts of the latent vector. These have dimensions equal to rank of the zero-lag covariance matrix of the fast part and slow part, respectively. We refer to them as the fast minimal static factor and the slow minimal static factor throughout the paper. It is worthwhile mentioning that by defining the static factor for the fast part and the slow part separately, the resultant static factor is not necessarily of dimension equal to the rank of the zero-lag covariance matrix of the whole (fast plus slow part) latent vector. The latter is due to the fact the even though components of slow minimal static factor (fast minimal static factor) are linearly independent from each other, they may linearly depend on the components of fast minimal static factor (slow minimal static factor).

In this paper, we first obtain the model associated with the static factor; we refer to this model as the static factor model. We then use the frequency domain approach to identify the system parameters of the static factor model from the spectral matrix of its associated blocked system. We finally show situations under which the parameters of the original underlying system can be obtained from the parameter matrices of the associated static factor model.

This paper is structured as follows. In the next section the main results of the paper are presented. First, in Subsection II-A, we illustrate a procedure for obtaining a system model associated with the static factor from the original underlying system. The blocking technique is then used to obtain the blocked system corresponding to the static factor model. It is demonstrated that the blocked system associated with the static factor model and the blocked system related to the original underlying system have the same pole characteristic while we study a relation between their zero properties in Subsection II-B. It is shown in Subsection II-B that tall blocked systems associated with the static factor model are generically zero-free. Then we use the zero-free property to obtain the parameter matrices of the blocked system associated with the static factor model using the well-known technique of spectral factorization. Since the spectral factorization only delivers the system parameters of the blocked system, we then show how the parameter matrices of the static factor model and ultimately those of the original underlying unblocked system can be obtained from the parameter matrices of the blocked system in Subsection II-D. Finally, concluding remarks are provided in Section III.

\section{MAin Results}

In this section we provide the main results of the current paper. First, we introduce the notion of the static factor. Then we use the well-known technique of blocking to transfer the multirate static factor model into a blocked linear timeinvariant system.

\section{A. Problem formulation}

We suppose that there exists an underlying stable system operating at the highest sample rate. Let the dynamics of this system be described by:

$$
\begin{aligned}
x_{t+1} & =A x_{t}+B u_{t}, \\
y_{t} & =C x_{t}+D u_{t},
\end{aligned}
$$

where $x_{t} \in \mathbb{R}^{n}, y_{t} \in \mathbb{R}^{l}$ and $u_{t} \in \mathbb{R}^{m}$ is the state, the output and the input, accordingly. For this system, $y_{t}$ exists for all $t$. However, we are interested in the situation where not every entry is measured for all $t$. In particular, we consider the case where $y_{t}$ has components that are observed at different rates. For simplicity, in this paper we consider a case where outputs are provided at two rates which we refer to as the fast rate and the slow rate.

Without loss of generality we decompose $y_{t}$ as $y_{t}=$ $\left[\begin{array}{c}y_{t}^{f} \\ y_{t}^{s}\end{array}\right]$ where $y_{t}^{f} \in \mathbb{R}^{l^{f}}$ is observed at all $t$, the fast part, and for some positive integer $N, y_{t}^{s} \in \mathbb{R}^{l^{s}}$ is observed at $t=0, N, 2 N, \ldots$, the slow part; also $l^{f}>0, l^{s}>0$ and $l^{f}+l^{s}=l$. Accordingly, we decompose $C$ and $D$ as:

$$
C=\left[\begin{array}{l}
C^{f} \\
C^{s}
\end{array}\right], D=\left[\begin{array}{l}
D^{f} \\
D^{s}
\end{array}\right]
$$

Thus, the multirate linear system corresponding to what is measured has the following dynamics:

$$
\begin{aligned}
x_{t+1} & =A x_{t}+B u_{t} \quad t=0,1,2, \ldots \\
y_{t}^{f} & =C^{f} x_{t}+D^{f} u_{t} \quad t=0,1,2, \ldots \\
y_{t}^{s} & =C^{s} x_{t}+D^{s} u_{t} \quad t=0, N, 2 N, \ldots
\end{aligned}
$$

Now, let us consider the zero-lag covariance matrices associated with the fast and the slow components of $y_{t}$

$$
\begin{aligned}
& \mathbb{E}\left[\begin{array}{ll}
y_{t}^{f} & \left.y_{t}^{f^{T}}\right] \triangleq R_{y}^{f}(0),
\end{array}\right. \\
& \mathbb{E}\left[y_{t}^{s} y_{t}^{s^{T}}\right] \triangleq R_{y}^{s}(0) \text {. }
\end{aligned}
$$

Let us suppose that $\operatorname{rank}\left(R_{y}^{f}(0)\right)=r^{f}$ and $\operatorname{rank}\left(R_{y}^{s}(0)\right)=r^{s}$. There exist full-column rank matrices $Q^{f} \in \mathbb{R}^{l^{f} \times r^{f}}$ and $Q^{s} \in \mathbb{R}^{l^{s} \times r^{s}}$ such that $R_{y}^{f}(0)=Q^{f} Q^{f^{T}}$ and $R_{y}^{s}(0)=Q^{s} Q^{s^{T}}$. Note that $Q^{f}$ is unique up to a right multiplication by an orthogonal matrix (the same holds for $\left.Q^{s}\right)$.

Define $z_{t}^{f}$ and $z_{t}^{s}$ as:

$$
\begin{aligned}
z_{t}^{f} & =\Xi^{f} y_{t}^{f}, \quad t=0,1,2, \ldots \\
z_{t}^{s} & =\Xi^{s} y_{t}^{s}, \quad t=0, N, 2 N, \ldots
\end{aligned}
$$

where $\Xi^{f}=\left(Q^{f^{T}} Q^{f}\right)^{-1} Q^{f^{T}}$ and $\Xi^{s}=\left(Q^{s^{T}} Q^{s}\right)^{-1} Q^{s^{T}}$. We refer to $z_{t}^{f}$ and $z_{t}^{s}$ as the fast minimal static factor 
and the slow minimal static factor, accordingly. Clearly $\operatorname{rank}\left(R_{y}^{f}(0)\right)=\operatorname{rank}\left(\mathbb{E}\left[z_{t}^{f} z_{t}^{f^{T}}\right]\right)=r^{f}$ and $\operatorname{rank}\left(R_{y}^{s}(0)\right)=$ $\operatorname{rank}\left(\mathbb{E}\left[z_{t}^{s} z_{t}^{s^{T}}\right]\right)=r^{s}$.

We have $E\left[\left(y_{t}^{f}-Q^{f} z_{t}^{f}\right)\left(y_{t}^{f}-Q^{f} z_{t}^{f}\right)^{T}\right]=0$ and $E\left[\left(y_{t}^{s}-\right.\right.$ $\left.\left.Q^{s} z_{t}^{s}\right)\left(y_{t}^{s}-Q^{s} z_{t}^{s}\right)^{T}\right]=0$ so,

$$
\begin{aligned}
& y_{t}^{f}=Q^{f} z_{t}^{f}, \\
& y_{t}^{s}=Q^{s} z_{t}^{s}
\end{aligned}
$$

We define the following state space dynamics associated with the fast and the slow minimal static factors:

$$
\begin{aligned}
x_{t+1} & =A x_{t}+B u_{t}, \quad t=0,1,2, \ldots \\
z_{t}^{f} & =C_{z}^{f} x_{t}+D_{z}^{f} u_{t}, \quad t=0,1,2, \ldots \\
z_{t}^{s} & =C_{z}^{s} x_{t}+D_{z}^{s} u_{t}, \quad t=0, N, 2 N, \ldots
\end{aligned}
$$

where,

$$
\begin{aligned}
C_{z}^{f} & =\Xi^{f} C^{f}, \\
D_{z}^{f} & =\Xi^{f} D^{f}, \\
C_{z}^{s} & =\Xi^{s} C^{s}, \\
D_{z}^{s} & =\Xi^{s} D^{s} .
\end{aligned}
$$

We refer to the system in (6) as the static factor model. The systems (6) and (2) are periodic in their output parameters. Thus, we use the blocking technique for working with these systems because this technique enables us to use the welldeveloped tools introduced in linear time-invariant systems analysis while dealing with blocked version of the systems (6) and (2). Moreover, some of the properties of the systems (6) and (2) such as zeros or poles are defined as those of their corresponding blocked versions [10].

We first specify the blocked system associated with the system (2). For $\tau \in\{1,2, \ldots, N\}$, we define

$$
\begin{aligned}
& Y_{t}^{\tau} \triangleq {\left[\begin{array}{c}
y_{t+\tau}^{f} \\
y_{t+\tau+1}^{f} \\
\vdots \\
y_{t+\tau+N-1}^{f} \\
y_{t+N}^{s}
\end{array}\right], } \\
& U_{t}^{\tau} \triangleq\left[\begin{array}{c}
u_{t+\tau} \\
u_{t+\tau+1} \\
\vdots \\
u_{t+\tau+N-1}
\end{array}\right], t=0, N, 2 N, \ldots \\
& x_{t}^{\tau} \triangleq x_{t+\tau}
\end{aligned}
$$

Then the associated blocked system is given by:

$$
\begin{aligned}
x_{t+N}^{\tau} & =\bar{A}_{\tau} x_{t}^{\tau}+\bar{B}_{\tau} U_{t}^{\tau}, \quad t=0,1,2, \ldots \\
Y_{t}^{\tau} & =\bar{C}_{\tau} x_{t}^{\tau}+\bar{D}_{\tau} U_{t}^{\tau}, \quad t=0, N, 2 N, \ldots
\end{aligned}
$$

where

$$
\begin{aligned}
& \bar{A}_{\tau} \triangleq A^{N}, \\
& \bar{B}_{\tau} \triangleq\left[\begin{array}{lllll}
A^{N-1} B & A^{N-2} B & \ldots & A B & B
\end{array}\right], \\
& \bar{C}_{\tau} \triangleq\left[C^{f^{T}} A^{T} C^{f^{T}} \ldots A^{(N-1)^{T}} C^{f^{T}} A^{(N-\tau)^{T}} C^{s^{T}}\right]^{T}, \\
& \bar{D}_{\tau} \triangleq\left[\begin{array}{cccc}
D^{f} & 0 & \ldots & 0 \\
C^{f} B & D^{f} & \ldots & 0 \\
\vdots & \vdots & \ddots & \vdots \\
C^{f} A^{N-2} B & C^{f} A^{N-3} B & \ldots & D^{f} \\
C^{s} A^{N-\tau-1} B & \ldots & D^{s} & *
\end{array}\right] .
\end{aligned}
$$

Moreover, in $\bar{D}_{\tau}$, * at the lower corner denotes $\tau-1$ zero matrices of size $l^{s} \times m$ and when $N-\tau-1<0, C^{s} A^{-1} B$ is replaced by $D^{s}$ and the rest of the terms in the last row are replaced by zero matrices of size $l^{s} \times m$.

Similarly, we denote the blocked system associated with the system (6) as:

$$
\begin{aligned}
x_{t+N}^{\tau} & =\hat{A}_{\tau} x_{t}^{\tau}+\hat{B}_{\tau} U_{t}^{\tau}, \quad t=0,1,2, \ldots \\
Z_{t}^{\tau} & =\hat{C}_{\tau} x_{t}^{\tau}+\hat{D}_{\tau} U_{t}^{\tau}, \quad t=0, N, 2 N, \ldots
\end{aligned}
$$

where

$$
Z_{t}^{\tau} \triangleq\left[\begin{array}{c}
z_{t+\tau}^{f} \\
z_{t+\tau+1}^{f} \\
\vdots \\
z_{t+\tau+N-1}^{f} \\
z_{t+N}^{s}
\end{array}\right], t=0, N, 2 N, \ldots
$$

and

$$
\begin{aligned}
& \hat{A}_{\tau} \triangleq A^{N} \\
& \hat{B}_{\tau} \triangleq\left[\begin{array}{lllll}
A^{N-1} B & A^{N-2} B & \ldots & A B & B
\end{array}\right] \\
& \hat{C}_{\tau} \triangleq \Phi \bar{C}_{\tau} \\
& \hat{D}_{\tau} \triangleq \Phi \bar{D}_{\tau} .
\end{aligned}
$$

Furthermore, $\Phi=\left[\begin{array}{cccc}\Xi^{f} & 0 & \ldots & 0 \\ & \ddots & & \vdots \\ 0 & & \Xi^{f} & 0 \\ 0 & & 0 & \Xi^{s}\end{array}\right]$. It is obvious that $\bar{A}_{\tau}=\hat{A}_{\tau}$ and $\bar{B}_{\tau}=\hat{B}_{\tau}$. For use in the rest of this paper, we define an operator $Z$ such that $Z x_{t}^{\tau}=x_{t+N}^{\tau}$, $Z U_{t}^{\tau}=U_{t+N}^{\tau}, Z Y_{t}^{\tau}=Y_{t+N}^{\tau}$ and $Z Z_{t}^{\tau}=Z_{t+N}^{\tau}$. The operator $Z$ is also used to denote a complex value.

\section{B. Zeros}

Let $\bar{\sum}_{\tau}$ and $\hat{\sum}_{\tau}$ denote the systems defined in (9) and (11), respectively. As mentioned earlier, $\bar{A}_{\tau}=\hat{A}_{\tau}$ which implies that the poles of two systems are identical. Thus, we focus on the zeros; in the rest of this part we study the relation between zeros of two systems i.e $\bar{\Sigma}_{\tau}$ and $\hat{\Sigma}_{\tau}$, and show when these systems are generically zero-free. To do this, let us first recall the definition of zeros for linear timeinvariant systems from [11] and [3]. 
Definition 2.1: The finite zeros of the system $\sum_{\tau}^{-}$are defined to be the finite values of $Z$ for which the rank of the following system matrix falls below its normal rank:

$$
\bar{M}_{\tau}(Z)=\left[\begin{array}{cc}
Z I-\bar{A}_{\tau} & -\bar{B}_{\tau} \\
\bar{C}_{\tau} & \bar{D}_{\tau}
\end{array}\right] .
$$

Furthermore, the system $\sum_{\tau}$ is said to have an infinite zero when $n+\operatorname{rank}\left(\bar{D}_{\tau}\right)$ is less than the normal rank of $\bar{M}_{\tau}(Z)$ or equivalently the rank of $\bar{D}_{\tau}$ is less than the normal rank of $\bar{V}_{\tau}(Z)=\bar{C}_{\tau}\left(Z I-\bar{A}_{\tau}\right)^{-1} \bar{B}_{\tau}+\bar{D}_{\tau}$.

Remark 2.2: Note that when the quadruple $\left\{\bar{A}_{\tau}, \bar{B}_{\tau}, \bar{C}_{\tau}, \bar{D}_{\tau}\right\}$ is a minimal realization of the transfer function $\bar{V}_{\tau}(Z)$, the zeros are just determined by the transfer function. Furthermore, they will coincide with those obtained from other well-known starting points for consideration of zeros e.g. Smith-McMillan form and coprime polynomial matrix fraction description. It is also worthwhile mentioning that when the realization is nonminimal, any uncontrollable and unobservable mode is also a zero.

In a completely analogous way we can define the zeros of the system $\hat{\Sigma}_{\tau}$.

We need to present the next two theorems from our previous works in [6] and [12].

Theorem 2.3: For a generic choice of matrices $\left\{A, B, C^{s}, C^{f}, D^{s}, D^{f}\right\} \quad$ with $l^{f}>m$. The system $\sum_{\tau}$ has no zeros.

Theorem 2.4: For a generic choice of matrices $\left\{A, B, C^{s}, C^{f}, D^{s}, D^{f}\right\}$ with $l^{f} \leq m$ and $N l^{f}+l^{s}>N m$. The system $\sum_{\tau}$ has no finite nonzero zeros.

The last two theorems studied the zero-freeness of $\sum_{\tau}$. We now explore the zero-freeness of $\hat{\sum}_{\tau}$.

Theorem 2.5: Consider the systems $\bar{\sum}_{\tau}$ and $\hat{\sum}_{\tau}$ defined above. Let $\hat{M}_{\tau}(Z)$ denote the system matrix associated with the system $\hat{\sum}_{\tau}$. Then the following equality holds:

$$
\operatorname{rank}(\bar{M}(Z))=\operatorname{rank}(\hat{M}(Z)) .
$$

Proof: The proof is omitted.

The following is then immediate.

Corollary 2.6: Consider the systems $\bar{\sum}_{\tau}$ and $\hat{\sum}_{\tau}$. Then $\bar{\Sigma}_{\tau}$ has a zero if and only if $\hat{\Sigma}_{\tau}$ has a zero.

Theorem 2.7: Consider a transfer function matrix $\bar{V}(Z)$ with minimal realization $\left\{\bar{A}_{\tau}, \bar{B}_{\tau}, \bar{C}_{\tau}, \bar{D}_{\tau}\right\}$. Suppose that the matrices $\left\{A, B, C^{s}, C^{f}, D^{s}, D^{f}\right\}$ assume generic values. Then

1) if $l^{f}>m$, the system $\hat{\sum}_{\tau}$ has no finite or infinite zeros.

2) if $l^{f} \leq m$ and $N l^{f}+l^{s}>N m$, the system $\hat{\sum}_{\tau}$ has no finite nonzero zeros.

Proof: The conclusion of the theorem becomes immediate in the light of Theorem 2.3, Theorem 2.4 and Corollary 2.6 .

In this subsection the main focus is on the zero properties of the systems $\sum_{\tau}, \hat{\Sigma}_{\tau}$. However, in the following theorem we study the minimality of the systems $\sum_{\tau}$ and $\hat{\Sigma}_{\tau}$; the conclusion of this theorem is important for the results which appear in the next subsection.
Theorem 2.8: Consider the multirate static factorsystem (6) defined by sextuple $\left\{A, B, C_{z}^{f}, D_{z}^{f}, C_{z}^{s}, D_{z}^{s}\right\}$ and its corresponding multirate system (2) defined by the sextuple $\{A, B, \underbrace{Q^{f} C_{z}^{f}}_{C^{f}}, \underbrace{Q^{f} D_{z}^{f}}_{D^{f}}, \underbrace{Q^{s} C_{z}^{s}}_{C^{s}}, \underbrace{Q^{s} D_{z}^{s}}_{D^{s}}\}$. Let $\hat{\Sigma}_{\tau}$ denote the blocked system associated with (6) defined by the quadruple $\left\{\hat{A}_{\tau}, \hat{B}_{\tau}, \hat{C}_{\tau}, \hat{D}_{\tau}\right\}$ and $\sum_{\tau}$ denote the blocked system associated with (2) defined by the quadruple $\left\{\bar{A}_{\tau}, \bar{B}_{\tau}, \bar{C}_{\tau}, \bar{D}_{\tau}\right\}$. Then, the system $\hat{\sum}_{\tau}$ is minimal if and only if the system $\sum_{\tau}$ is minimal.

Proof: The proof is omitted.

\section{Spectral factorization}

In the previous subsection, it is shown that the system $\hat{\sum}_{\tau}$ is generically zero-free. In this subsection we use this property to obtain the parameter matrices associated with $\sum_{\tau}$ from its corresponding real rational spectral matrix.

In general, consider a square real rational matrix function $\hat{\Phi}_{\tau}(Z)$ where $\hat{\Phi}_{\tau}(Z)=\hat{\Phi}_{\tau}^{T}\left(Z^{-1}\right)$ with $\hat{\Phi}_{\tau}\left(e^{j \omega}\right)$ being bounded and nonnegative definite for $\omega \in[-\pi, \pi]$. We refer to $\hat{\Phi}_{\tau}(Z)$ as the spectral matrix. Let $\hat{\Phi}_{\tau}(Z)$ be a spectral matrix of size $\left(N r^{f}+r^{s}\right) \times\left(N r^{f}+r^{s}\right)$ with normal rank $N m$. Then there exists a $\left(N r^{f}+r^{s}\right) \times N m$ real rational spectral factor, call it $\tilde{V}_{\tau}(Z)$, such that $\hat{\Phi}_{\tau}(Z)=\tilde{V}_{\tau}(Z) \tilde{V}_{\tau}^{T}\left(Z^{-1}\right)$ and $\tilde{V}_{\tau_{\tilde{V}}}(Z)$ has no poles in $|Z| \geq 1$, including infinity, $\operatorname{rank}\left(\tilde{V}_{\tau}(Z)\right)=N m$ in $|Z|>1$ including infinity. Furthermore, if $\hat{\Phi}_{\tau}(Z)$ has rank $N m$ on $|Z|=1$ then $\tilde{V}_{\tau}(Z)$ has constant rank $N m$ in $|Z| \geq 1$. The transfer function $\tilde{V}_{\tau}(Z)$ is sometimes referred to as the stable minimum phase spectral factor. Note that $\tilde{V}_{\tau}(Z)$ is unique up to post multiplication by an orthogonal constant matrix [13].

We now seek to apply this knowledge to a particular spectrum, namely that resulting from the output of the system $\hat{\sum}_{\tau}$ when it is driven by a white noise input of unit covariance. With a slight abuse of notation we denote this spectrum by $\hat{\Phi}_{\tau}(Z)$ which is in fact expressible in the form:

$$
\hat{\Phi}_{\tau}(Z)=\hat{R}_{\tau}+\hat{C}_{\tau}\left(Z I-\hat{A}_{\tau}\right)^{-1} \hat{K}_{\tau}+\hat{K}_{\tau}^{T}\left(Z^{-1} I-\hat{A}_{\tau}^{T}\right)^{-1} \hat{C}_{\tau}^{T}
$$

where,

$$
\begin{gathered}
\hat{R}_{\tau}=\hat{C}_{\tau} \hat{P}_{\tau} \hat{C}_{\tau}^{T}+\hat{D}_{\tau} \hat{D}_{\tau}^{T}, \\
\hat{K}_{\tau}=\hat{A}_{\tau} \hat{P}_{\tau} \hat{C}_{\tau}^{T}+\hat{B}_{\tau} \hat{D}_{\tau}^{T}, \\
\hat{P}_{\tau}=\hat{A}_{\tau} \hat{P}_{\tau} \hat{A}_{\tau}^{T}+\hat{B}_{\tau} \hat{B}_{\tau}^{T},
\end{gathered}
$$

and $\hat{P}_{\tau}=\mathbb{E}\left[x_{N t}^{\tau} x_{N t}^{\tau}{ }^{T}\right]$.

Our interest is in determining what $\hat{\sum}_{\tau}$ is, given knowledge of $\hat{\Phi}_{\tau}(Z)$. To this end, we make the following assumptions about $\hat{\sum}_{\tau}$, but without assuming we know its transfer function or state variable description: $\hat{\sum}_{\tau}$ is stable, linear time-invariant finite-dimensional system driven by a white noise input of unit covariance. (An additional assumption will be made below concerning the zero properties of the system). 
The assumptions on $\hat{\sum}_{\tau}$ guarantee that $\hat{\Phi}_{\tau}(Z)$ is a real rational spectral matrix and expressible in the form of (15) where $\left\{\hat{A}_{\tau}, \hat{C}_{\tau}, \hat{K}_{\tau}\right\}$ is reachable and observable. This means that $\hat{\sum}_{\tau}$ has a state variable description in the form $\left\{\hat{A}_{\tau}, \hat{B}_{\tau}, \hat{C}_{\tau}, \hat{D}_{\tau}\right\}$, i.e. with the same $\hat{A}_{\tau}, \hat{C}_{\tau}$, and with $\hat{B}_{\tau}$, $\hat{D}_{\tau}$ and a further matrix $\hat{P}_{\tau}$ satisfying (16).

In general, (16) is difficult to solve for the unknown matrices. Nevertheless, given a zero-free property, solution is straightforward. To understand this we state some important results initially introduced in [13] which relate the zerofree property of $\hat{\sum}_{\tau}$ to its associated spectral matrix. The definition of zeros for the spectral matrix $\hat{\Phi}_{\tau}(Z)$ is stated in the following.

Definition 2.9: The spectral factor $\hat{\Phi}_{\tau}(Z)$ has a zero at $Z_{0}$, where $Z_{0} \in \mathbb{C}-\{0\}$, if and only if the following matrix has rank less than its normal rank at $Z_{0}$

$$
M_{\hat{\Phi}_{\tau}}(Z)\left[\begin{array}{ccc}
Z I-\hat{A}_{\tau} & 0 & -\hat{K}_{\tau} \\
0 & Z^{-1} I-\hat{A}_{\tau}^{T} & -\hat{C}_{\tau}^{T} \\
\hat{C}_{\tau} & \hat{K}_{\tau} & \hat{R}_{\tau}
\end{array}\right]
$$

Furthermore, $\hat{\Phi}_{\tau}(Z)$ has a zero at infinity and at zero if and only if the following matrix has rank less than $n+N m$

$$
N_{\tau}=\left[\begin{array}{cc}
-\hat{A}_{\tau} & -\hat{K}_{\tau} \\
\hat{C}_{\tau} & \hat{R}_{\tau}
\end{array}\right]
$$

Lemma 2.10: Consider the system $\hat{\sum}_{\tau}$ with minimal realization $\left\{\hat{A}_{\tau}, \hat{B}_{\tau}, \hat{C}_{\tau}, \hat{D}_{\tau}\right\}$ and transfer function $\hat{V}_{\tau}(Z)$. Suppose that all eigenvalues of $\hat{A}_{\tau}$ lie within the unit circle, and $u_{t}$ is a zero mean, white noise with unit covariance matrix applied to the system $\hat{\sum}_{\tau}$ from time $-\infty$. Let the $\hat{\Phi}_{\tau}(Z)$ be the associated power spectrum which is defined by (15) using (16). Then the system $\hat{\sum}_{\tau}$ has no zeros in $\mathbb{C} \cup\{\infty\}$, if and only if $\hat{\Phi}_{\tau}(Z)$ has no zeros in $\mathbb{C} \cup\{\infty\}$.

We remark the transfer function $\hat{V}_{\tau}(Z)$ of the previous lemma is a minimum phase spectral factor of $\hat{\Phi}_{\tau}(Z)$, as can be seen from the earlier definition of such a spectral factor and the assumed properties of $\hat{V}_{\tau}(Z)$.

In addition, the following result illustrates that if the spectral matrix is zero-free then the parameter matrices of the associated system can be computed in a finite number of rational calculations using the spectral factorization technique, see [13]. Generally, determination of the minimum phase spectral factor of a spectral matrix cannot be achieved by rational calculation; thus the zero-free property implies great simplification.

Lemma 2.11: Suppose that the spectral matrix $\hat{\Phi}_{\tau}(Z)$ is generated from the system $\hat{\sum}_{\tau}$. Also, assume that $\hat{\sum}_{\tau}$ is minimal and zero-free in $\mathbb{C} \cup\{\infty\}$. Then there exists a unique matrix $\hat{P}_{\tau}$ obtainable in a finite number of rational calculations from the matrices $\hat{A}_{\tau}, \hat{K}_{\tau}, \hat{C}_{\tau}$ and $\hat{R}_{\tau}$, so that the state space representation for $\hat{\sum}_{\tau}$ can be determined. Moreover, the matrices $\hat{B}_{\tau}$ and $\hat{D}_{\tau}$ can only be obtained up to post multiplication by a constant orthogonal matrix. Formal rigorous proofs of the above lemmas can be found in [13].
The conclusion of the above lemma states the parameter matrices $\hat{D}_{\tau}$ and $\hat{B}_{\tau}$ can be obtained from $\hat{\Phi}_{\tau}(Z)$ and are only unique up to an orthogonal post multiplication. Furthermore, the matrix $\hat{D}_{\tau}$ which satisfies (11) has to have a certain structure, see (13). The following theorem establishes a relation between different $\hat{D}_{\tau}$ and $\hat{B}_{\tau}$.

Theorem 2.12: Let two stable minimum phase spectral factors with the same number of columns, say $\hat{V}_{\tau}^{1}(Z)$ and $\hat{V}_{\tau}^{2}(Z)$, associated with the same spectral matrix $\hat{\Phi}_{\tau}(Z)$ be defined by the quadruples $\hat{A}_{\tau}, \hat{B}_{\tau}^{1}, \hat{C}_{\tau}, \hat{D}_{\tau}^{1}$ and $\hat{A}_{\tau}$, $\hat{B}_{\tau}^{1} J=\hat{B}_{\tau}^{2}, \hat{C}_{\tau}, \hat{D}_{\tau}^{1} J=\hat{D}_{\tau}^{2}$. Suppose that both $\hat{D}_{\tau}^{2}$ and $\hat{D}_{\tau}^{1}$ are block lower triangular matrices and diagonal blocks in both matrices have full column rank. Suppose further that $\hat{D}_{\tau}^{1}$ has the same diagonal structure as $\hat{D}_{\tau}$ in (11). Then the orthogonal matrix $J$ is the direct sum of $N+1$ orthogonal matrices $J_{i i}, i \in\{1, \ldots, N+1\}$, with the block sizes equal to the number of columns in the diagonal blocks in $\hat{D}_{\tau}^{1}$.

Proof: The proof is omitted.

\section{From the parameter matrices of blocked systems to parameter matrices of the associated unblocked systems}

The previous subsection studied the spectral factorization of the system $\hat{\sum}_{\tau}$. It argued that generically the spectral matrix associated with a tall blocked system is zero-free and so the parameter matrices of the blocked system can be obtained using a finite number of rational calculations, up to a certain orthogonal multiplier. One should note that even though the parameter matrices of $\hat{\sum}_{\tau}$ may be known, using this information to obtain the parameter matrices of the associated unblocked system is a nontrivial task. Thus, in this part, we use the results of the previous section and assume that the parameter matrices associated with $\hat{\sum}_{\tau}$ are available with the intention is of using these parameter matrices to obtain the system parameters of the system (6). In the following, we first provide a theorem which states that the parameter matrices associated with the static factor model can be recovered from the parameter matrices of its corresponding blocked system $\hat{\sum}_{\tau}$. Then we illustrate how the parameter matrices of the original underlying system can be obtained from the parameter matrices of the associated static factor model.

Theorem 2.13: Suppose the parameter matrices of the system $\hat{\sum}_{\tau}$ i.e. $\hat{A}_{\tau}, \hat{B}_{\tau}, \hat{C}_{\tau}, \hat{D}_{\tau}$, are available and the pairs $\left(\hat{A}_{\tau}, \hat{B}_{\tau}\right)$ and $\left(\hat{A}_{\tau}, \hat{C}_{\tau}\right)$ are reachable and observable. Then from the matrices $\hat{A}_{\tau}, \hat{B}_{\tau}, \hat{C}_{\tau}, \hat{D}_{\tau}$, the associated parameter matrices for the unblocked system i.e. $A, B, C^{z}, D^{z}$, can be determined.

Proof: The proof is omitted.

With the help of the above theorem the parameters $A, B$, $C^{z}$ and $D^{z}$ become available. Note that both systems (6) and (2) have the same $A$ and $B$ matrices; by using the equation (5), one can easily obtain the parameter matrices associated with measured outputs of the original underlying systems as: 


$$
\begin{aligned}
& C^{f}=Q^{f} C_{z}^{f}, \\
& D^{f}=Q^{f} D_{z}^{f}, \\
& C^{s}=Q^{s} C_{z}^{s}, \\
& D^{s}=Q^{s} D_{z}^{s} .
\end{aligned}
$$

Hence, all parameter matrices of the original underlying system can be recovered.

\section{CONCLUSIONS}

In this paper we studied tall systems whose outputs are measured at different rates. In particular, the case of two rates of outputs, the fast outputs and the slow outputs, was considered. In particular, we used the notion of the static factor to deal with the case when these systems have measurements with an extremely large cross-sectional dimension. We then obtained the model associated with the static factor, the static factor model, which is periodic in its output parameters. Hence, we used the well-known technique of blocking to obtain the blocked system associated with the former model. It was illustrated that tall blocked systems are generically zero-free. Then some important results from [13] on the spectral factorization of linear time-invariant systems were recalled to help us to obtain the parameter matrices associated with the blocked system. We then used the parameter matrices associated with the blocked system to obtain the parameter matrices involved with the static factor model. Finally, we recovered all parameter matrices of the original underlying system.

\section{ACKNOWLEDGEMENTS}

Support by the ARC Discovery Project Grant DP1092571, by the FWF (Austrian Science Fund) under contracts P17378 and P20833/N18 and by the Oesterreichische Forschungsgemeinschaft is gratefully acknowledged.

\section{REFERENCES}

[1] M. Forni, M. Hallin, M. Lippi, and L. Reichlin, "The generalized dynamic-factor model: Identification and estimation," The Review of Economics and Statistics, vol. 82, no. 4, pp. 540-554, November 2000.

[2] B. D. O. Anderson, M. Zamani, and G. Bottegal, "On the zero properties of tall linear systems with single-rate and multirate outputs," in Mathematical System Theory - Festschrift in Honor of Uwe Helmke on the Occasion of his Sixtieth Birthday, K. Hüper and J. Trumpf, Eds. CreateSpace, 2013, pp. 31-49.

[3] T. Kailath, Linear systems. Prentice-Hall, New Jersey, 1980.

[4] A. Filler, "Generalized dynamic factor models structure theory and estimation for single frequency and mixed frequency data," Ph.D. dissertation, Vienna University of Technology, 2010.

[5] M. Deistler, B. D. O. Anderson, A. Filler, C. Zinner, and W. Chen, "Generalized linear dynamic factor models: An approach via singular autoregressions," European Journal of Control, vol. 16, pp. 211-224, 2010.

[6] M. Zamani and B. D. O. Anderson, "On the zero properties of linear discrete-time systems with multirate outputs," in Proceedings of American Control Conference (ACC), 2012, pp. 5182-5187.

[7] B. D. O. Anderson, M. Deistler, E. Felsenstein, B. Funovits, P. Zadrozny, M. Eichler, W. Chen, and M. Zamani, "Identifiability of regular and singular multivariate autoregressive models from mixed frequency data," in Proceedings of the IEEE Conference on Decision and Control, 2012, pp. 184-189.

[8] B. Chen and P. A. Zadrozny, "An extended Yule-Walker method for estimating a vector autoregressive model with mixed-frequency," Advances in Econometrics, vol. 13, pp. 47-73.
[9] M. Zamani, E. Felsenstein, M. Deistler, and B. D. O. Anderson, "Mixed frequency structured AR model identification," in Proceedings of the European Control Conference, to appear.

[10] S. Bittanti and P. Colaneri, Periodic Systems Filtering and Control, ser. Communications and Control Engineering. Springer-Verlag, 2009.

[11] J. P. Hespanha, Linear systems theory. Princton University Press, 2009.

[12] M. Zamani, W. Chen, B. D. O. Anderson, M. Deistler, and A. Filler, "On the zeros of blocked linear systems with single and mixed frequency data," in Proceedings of the IEEE Conference on Decision and Control and European Control Conference (CDC-ECC), 2011, pp. 4312-4317.

[13] B. D. O. Anderson and M. Deistler, "Properties of zero-free spectral matrices," IEEE Transactions on Automatic Control, vol. 54, no. 10, pp. $2365-2375,2009$. 